\title{
Cable installation simulation by using a multibody dynamic model
}

\author{
Cai Jin Yang • Di Feng Hong • Ge Xue Ren • \\ Zhi Hua Zhao
}

Received: 20 February 2012 / Accepted: 3 April 2013 / Published online: 23 April 2013

(C) The Author(s) 2013. This article is published with open access at Springerlink.com

\begin{abstract}
A major concern when installing the cables into the underground conduit is minimizing the tensile forces exerted on the cables as they are pulled. This knowledge makes it possible to avoid over conservative design practices and to achieve substantial saving during construction. A general computing algorithm of predicting the tensile force of the cable pulled through the underground conduit with an arbitrary configuration is presented in this paper, which is based on multibody system dynamic formulation. The presented multibody dynamic model for this problem consists of the cable, the underground conduit, and the interaction between the cable and the conduit. In this paper, the cable is modeled by the finite cable element based on an absolute nodal coordinate formulation. The interaction between the cable and the underground conduit is described by the Hertz contact theory. Numerical examples are presented to illustrate the effectiveness and efficiency of the proposed method for estimating the cable tension.
\end{abstract}

Keywords Cable installation $\cdot$ Tension $\cdot$ Pulled $\cdot$ Multibody dynamic model $\cdot$ Hertz contact

\section{Introduction}

Nowadays, power cables, fiber optic cables, and other in-building communication cables are often placed in the underground conduit. For the cable underground installation, the conduit protects the cables from shifting rocks, aggressive rodents, and/or damage from hand shovels. Moreover, the cables in the conduit are replaced or upgraded easily. The old cables can be drawn out from the conduit, and the new ones can be pulled into the conduit without extensive and expensive digging.

The tensile force exerted on the cables when they are pulled in the conduit system is a major concern in cable installation. Excessive tension can damage the cables and cause the failure or deterioration of the cables. Accurate prediction of the tensile force of the cable is essential for the design of the conduit system. For example, the big petroleum industry

C.J. Yang $(\bowtie) \cdot$ D.F. Hong · G.X. Ren · Z.H. Zhao

Department of Engineering Mechanics, Tsinghua University, Beijing, 100084, China

e-mail: ycj78_2012@163.com 
nowadays has large underground electrical systems to provide electrical power. When designing the underground electrical distribution systems, the limiting factors imposed by the design criteria of tensile force should be considered.

Studies have so far focused on the methods for predicting the tensile force of the cable, especially the maximum tensile force that the cables can tolerate safely during the cable installation. The analytical model available to date for predicting the tensile forces of the cables in the conduit is that proposed by R.C. Rifenburg [1] and generalized by D.G. Smith [2]. This classical model treats the cable as a very flexible rope without bending stiffness, considering the cable weight and the additional longitudinal friction against the intrados of the bends, related to the change in the direction of the cable tension. But if the cable has complex spatial geometry, the contribution of bending deformation to the tension exerted on the cable must be considered. In this situation, such analytical model is now not valid. In 1996, Mircea Iordanescu [3] presented an analytical model incorporating the bending stiffness of the cable to predict the maximum pulling force exerted on the cable during the cable installation. This model also considers the geometrical and physical nonlinearity of the behavior of the cable in bending and subjected to high tension. However, this model was originally developed for the bending conduit system and gave a conservative estimation for the tensile force of the cable in the straight segment of the conduit system. Several other formulas [4-7], for predicting the cable tension have also been proposed: some formulas are simple in an approximate manner, and some formulas are complex and exact but only for the specific cases.

The objective of this study is to develop a general computing algorithm of predicting the tensile force of the cable when pulled into the conduit of arbitrary configuration. To achieve this goal, a multibody dynamic model composed of a flexible cable, the rigid conduit and the contact between the cable and the underground conduit is presented in this paper. The cable is modeled by the finite cable element based on an absolute nodal coordinate formulation [8]. The interaction model between the cable and the conduit is described by the Hertz contact theory, which captures the friction and contact between the cable and the conduit.

This paper is organized as follows. In Sect. 2, a multibody dynamic model for predicting the tensile force of the cable pulled in the underground conduit is presented. The theory of the finite cable element is first briefly described and the contact model between the cable and the conduit is followed. An effective contact check technique is also presented to improve the efficiency of the computation and be implemented in this model. Four illustrative examples are examined in the third section to verify the effectiveness of the proposed method. Conclusions are included in the last section.

\section{A multibody dynamic model for cable installation}

A cable installation system, as shown in Fig. 1, is a typical multibody system, mainly consisting of a pulling rig, the cable and the conduit. In this section, multibody system dynamic formulation is applied to model the cable installation.

\subsection{Pulling rig}

In cable installation, the pulling rig often provides a driven force, which makes the cable move through the conduit with a constant low speed. In this meaning, the pulling rig can be modeled as a force element. However, the accurate force model for the pulling rig is not generally available. Alternatively, an equivalent dynamic model for it can be described by a velocity constraint and is written as 


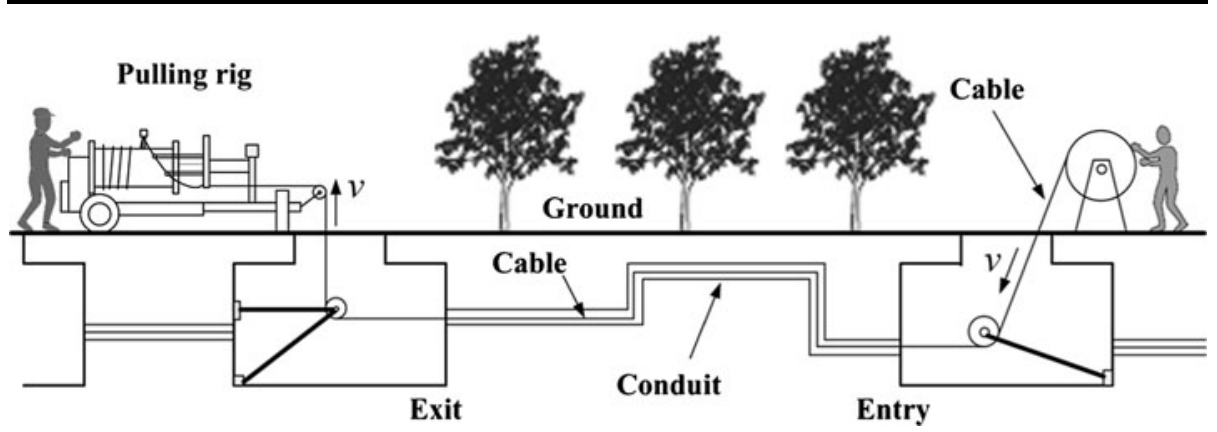

Fig. 1 Schematic of the cable installation

$$
\mathbf{C}_{d}\left(\mathbf{q}_{d}, \dot{\mathbf{q}}_{d}, t\right)=0
$$

where $\mathbf{C}_{d}$ is the constraint equation, $\mathbf{q}_{d}$ is the vector of generalized coordinate of the pulling rig. $\dot{\mathbf{q}}_{d}$ is the derivative of the generalized coordinate vector with respect to time $t$.

Because the constraint is directly exerted on the cable, $\mathbf{q}_{d}$ can be replaced by the generalized coordinate vector of the end node of the cable. The cable element is described in the following.

\subsection{Cable model}

As illustrated in Fig. 1, the cable is usually placed in parallel with the slope of the conduit at entry so that it can be smoothly pulled into the conduit. A straight cable element adapted from a three-dimensional Euler-Bernoulli element originally developed by Von Dombrowski [9] is used to model the cable in this section. The used cable element has the advantage of less degrees of freedom compared with the original ANCF elements $[10,11]$.

\subsubsection{The description of the cable element}

Figure 2 depicts the cable element in the initial and deformed configuration. As shown in Fig. 2, the configuration of the cable element is determined by the positions and the slopes of two end nodes, by which it can be coupled to and interact with other elements. The positions of the end nodes $i$ and $j$, are given by their absolute displacement vectors $\mathbf{r}_{i}$ and $\mathbf{r}_{j}$ in a global inertial system. The slopes $\mathbf{r}_{i}^{\prime}$ and $\mathbf{r}_{j}^{\prime}$ of the end nodes $i$ and $j$, are the derivative of their positions with respect to arc length $l$.

In an absolute coordinate formulation, the global position of a representative point $P$ on the centerline of the cable element at $l$ defined in the initial straight configuration can be expressed by

$$
\mathbf{r}_{P}=\mathbf{S}_{e} \mathbf{q}_{e}
$$

where $\mathbf{q}_{e}$ is the nodal coordinate vector of the cable element, which is defined by

$$
\mathbf{q}_{e}=\left[\begin{array}{llll}
\mathbf{r}_{i}^{T} & \mathbf{r}_{i}^{\prime T} & \mathbf{r}_{j}^{T} & \mathbf{r}_{j}^{\prime T}
\end{array}\right]^{T},
$$

and $\mathbf{S}_{e}$ is the element shape function, which is given by 
Fig. 2 The cable element

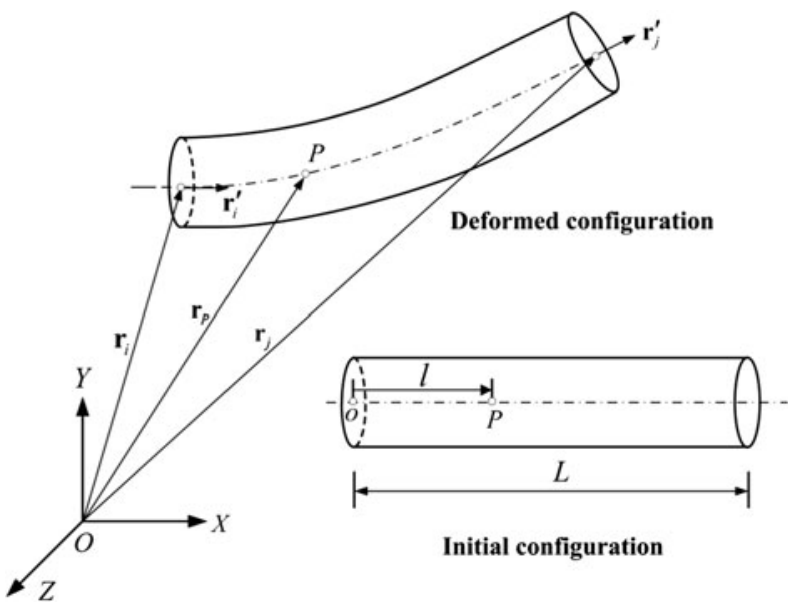

$$
\mathbf{S}_{e}=\left[\begin{array}{cccccccccccc}
N_{1} & 0 & 0 & N_{3} & 0 & 0 & N_{2} & 0 & 0 & N_{4} & 0 & 0 \\
0 & N_{1} & 0 & 0 & N_{3} & 0 & 0 & N_{2} & 0 & 0 & N_{4} & 0 \\
0 & 0 & N_{1} & 0 & 0 & N_{3} & 0 & 0 & N_{2} & 0 & 0 & N_{4}
\end{array}\right]
$$

in which the interpolation functions $N_{i}$ for $i=1,2,3,4$ are expressed as

$$
\begin{array}{ll}
N_{1}=(s-1)^{2}(s+2) / 4, & N_{3}=(s-1)^{2}(s+1) \cdot \frac{L}{8} \\
N_{2}=(s+1)^{2}(2-s) / 4, & N_{4}=(s+1)^{2}(s-1) \cdot \frac{L}{8}
\end{array}
$$

with the nondimensional coordinate

$$
s=\frac{2 l}{L}-1
$$

and $L$ is the initial length of the cable element.

\subsubsection{The kinetic energy of the cable element}

The velocity of the representative point $P$ on the centerline of the cable element can be expressed by

$$
\dot{\mathbf{r}}_{P}=\mathbf{S}_{e} \dot{\mathbf{q}}_{e}
$$

Electric cables usually have a small radius. So, the torsional effect of the cross-sections of the cable element is neglected in this paper. In this way, the cable element can be treated as an elastic line. Hence, the kinetic energy of the cable element can be simply decided by

$$
T=\frac{1}{2} \int_{0}^{L} \rho A \dot{\mathbf{r}}_{p} \cdot \dot{\mathbf{r}}_{p} d l=\frac{1}{2} \int_{0}^{L} \rho A\left(\mathbf{S}_{e} \dot{\mathbf{q}}_{e}\right) \cdot\left(\mathbf{S}_{e} \dot{\mathbf{q}}_{e}\right) d l
$$

where $\rho$ is the material density of the cable element and $A$ is the area of the circular crosssection of the cable element. 


\subsubsection{The strain energy of the cable element}

Because of neglecting the torsional effects of the cross-sections of the cable element, the strain energy $U$ of the cable element can be written in a simple form using two variables representing the bending curvature $\kappa$ and the longitudinal strain $\varepsilon$ of the cable axis:

$$
U=\frac{1}{2} \int_{0}^{L}\left(E A \varepsilon^{2}+E J \kappa^{2}\right) d l
$$

where $E$ is Young's modulus and $J$ is the inertia moment of the cross-section of the cable element.

The longitudinal strain $\varepsilon$ is defined by

$$
\varepsilon=\sqrt{\mathbf{r}_{p}^{\prime} \cdot \mathbf{r}_{p}^{\prime}}-1
$$

and the bending curvature $\kappa$ is given by

$$
\kappa=\frac{\left\|\mathbf{r}_{p}^{\prime} \times \mathbf{r}_{p}^{\prime \prime}\right\|}{\left\|\mathbf{r}_{p}^{\prime}\right\|^{3}}
$$

where $\|\cdot\|$ denotes the module of the vector. It is worthy of noting that longitudinal strain defined by Eq. (11) is valid as the radius of the cable element is small. If the cables have large radius, the bending-related strain [12] will be coupled in axial deformation. Details refer to literature [12].

\subsubsection{The motion equation of the cable element}

Based on the first Lagrange formulation, the motion equation of the cable element can be expressed by

$$
\left\{\begin{array}{l}
\frac{d}{d t}\left(\frac{\partial T}{\partial \dot{\mathbf{q}}_{e}}\right)-\frac{\partial(T-U)}{\partial \mathbf{q}_{e}}=\mathbf{Q}_{\mathbf{e}}+\left(\mathbf{C}_{\mathbf{q}_{e}}^{e}\right)^{T} \lambda_{e} \\
\mathbf{C}^{e}\left(\mathbf{q}_{e}, \dot{\mathbf{q}}_{e}, t\right)=0
\end{array}\right.
$$

where $\mathbf{C}^{e}$ is the constraint equation of the cable element. $\mathbf{C}_{\mathbf{q}_{e}}^{e}$ is the Jacobian matrix of the constraint. The superscript " $T$ " denotes the transpose matrix, $\lambda_{e}$ is Lagrange multipliers of the cable element. $\mathbf{Q}_{\mathbf{e}}$ is the generalized force vector of the cable element.

In the above model of the cable element, we also consider the viscoelasticity damping accounting for energy dissipation in the cable element. Here, we follow the internal damping model for the absolute nodal coordinate formulation in the cable element. More details are found in [13].

\subsection{Conduit model}

The distribution of the underground conduit is usually very complex. However, it mainly includes straight segment and bending segment. For a general purpose, the conduit with an arbitrary shape is considered in this paper. For the conduit with complex shape, it can be regard as the assembly of multiple straight segments, seen in Fig. 3. Each straight segment is a hollow cylinder, and the inner radius of the hollow is identical with the radius of the conduit.

Each straight segment is decided by two end points $P_{i}$ and $P_{j}$ on its axis. The positions of the end points $P_{i}$ and $P_{j}$ are given by the displacement vector $\mathbf{r}_{i}$ and $\mathbf{r}_{j}$ in the global coordinate system $O X Y Z$. The position data of these spatial points $P_{i}$ and $P_{j}$ can be obtained from the survey in the design of the conduit system. 


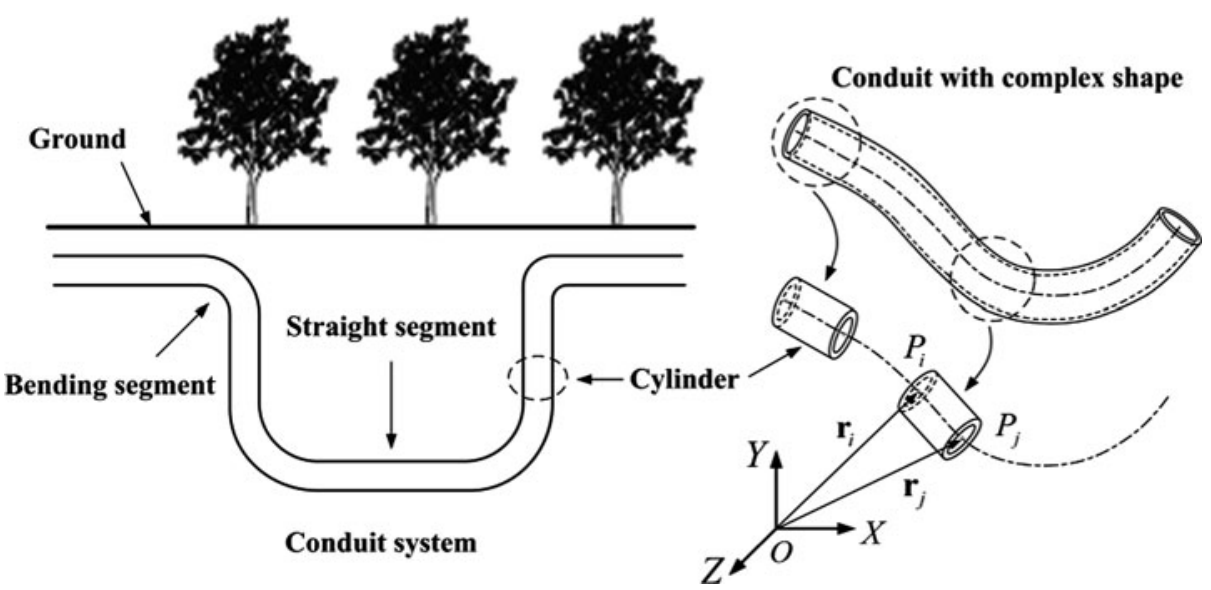

Fig. 3 The conduit model

Fig. 4 Contact model between the cable and the conduit

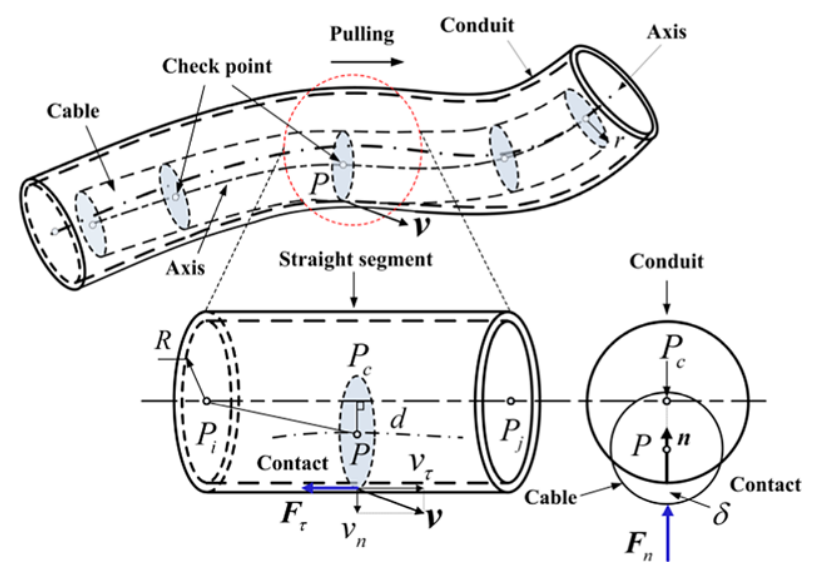

2.4 Contact model between the cable and the conduit

In installation, the cable is constrained by the conduit through the friction and contact. The contact dynamics involving the beam/cable with large slender ratio are very complex. Previous reports are very limited, e.g., [14]. In [14], an effective model for the contact between the beam and a circular surface was proposed. However, the application of this contact model to our work is unfeasible due to the complex profile of an actual conduit. Here, we follow the ideas of contact test used in [14] and present a more general method to model the contact between the cable and the conduit. To do it, some simplifications are made. Generally, the conduit is in a three-dimensional space. An approximate model for the conduit is found in the above text. To fit into the reduced model of the conduit, we further give an approximate model for the cable. Figure 4 shows the contact model between the cable and the conduit.

As illustrated in Fig. 4, we set a number of check points on the centerline of the cable element. These points are avail of performing contact test. The global position $\mathbf{r}_{p}$ of a representative check point $P$ on the centerline of the cable element is decided by Eq. (2). 
Based on the theory of spatial geometry and algebra, one can easily obtain the distant $d$ of the check point $P$ to the line segment $P_{i} P_{j}$. The point $P_{c}$ is the projection point of the check point $P$ onto the line $P_{i} P_{j}$. If the distant $d$ is greater than the clearance between the cable and the conduit and the point $P_{c}$ is on the line segment $P_{i} P_{j}$, then the cross-section of the cable in which the check point $P$ is, will contact with the inner wall of the conduit.

If the contact occurs, the depth of penetration $\delta$ is given by

$$
\delta=d-(R-r)
$$

where $R$ denotes the inner radius of the conduit, $r$ denotes the radius of the cross-section of the cable. The contact normal vector $\mathbf{n}$ is approximately decided by

$$
\mathbf{n}=\frac{\mathbf{P P}_{c}}{\left\|\mathbf{P P}_{c}\right\|}
$$

Let $\mathbf{v}$ be the velocity of contact point. Then the normal approaching velocity $v_{n}$ can be expressed as

$$
v_{n}=\mathbf{v} \cdot \mathbf{n}
$$

The tangential velocity $\mathbf{v}_{\tau}$ can be given by

$$
\mathbf{v}_{\tau}=\mathbf{v}-v_{n} \cdot \mathbf{n}
$$

Thus, the contact normal force $f_{n}$ is given by nonlinear Hertz contact law [15]

$$
f_{n}=K_{n} \delta^{e}+C_{n} \dot{\delta}
$$

where $K_{n}$ is the normal contact stiffness, $C_{n}$ is the contact damping coefficient, $e$ is the nonlinear exponent. $\dot{\delta}$ is the time rate of the change of penetration depth.

Once the normal contact force is determined, the tangent friction force $f_{\tau}$ is often characterized by Coulomb model

$$
f_{\tau}=\mu \cdot f_{n}
$$

where $\mu$ is the friction coefficient. The friction coefficient $\mu$ between the cable and the conduit is selected by engineering experiences or obtained from experimental data. For more details, one can refer to the literatures $[16,17]$.

The unit vector $\boldsymbol{\tau}$ of the friction force is decided by

$$
\boldsymbol{\tau}=\frac{\mathbf{v}_{\tau}}{\left\|\mathbf{v}_{\tau}\right\|}
$$

The resultant contact force $\mathbf{F}$ can be expressed by

$$
\mathbf{F}=\mathbf{F}_{n}+\mathbf{F}_{\tau}
$$

where the normal contact force $\mathbf{F}_{n}$ is given by

$$
\mathbf{F}_{n}=f_{n} \cdot \mathbf{n}
$$

and the tangential friction force $\mathbf{F}_{\tau}$ is given by

$$
\mathbf{F}_{\tau}=-f_{\tau} \cdot \boldsymbol{\tau}
$$

After considering the contact for all check points set in the cable element, one can obtain the total normal contact and tangential friction force. 


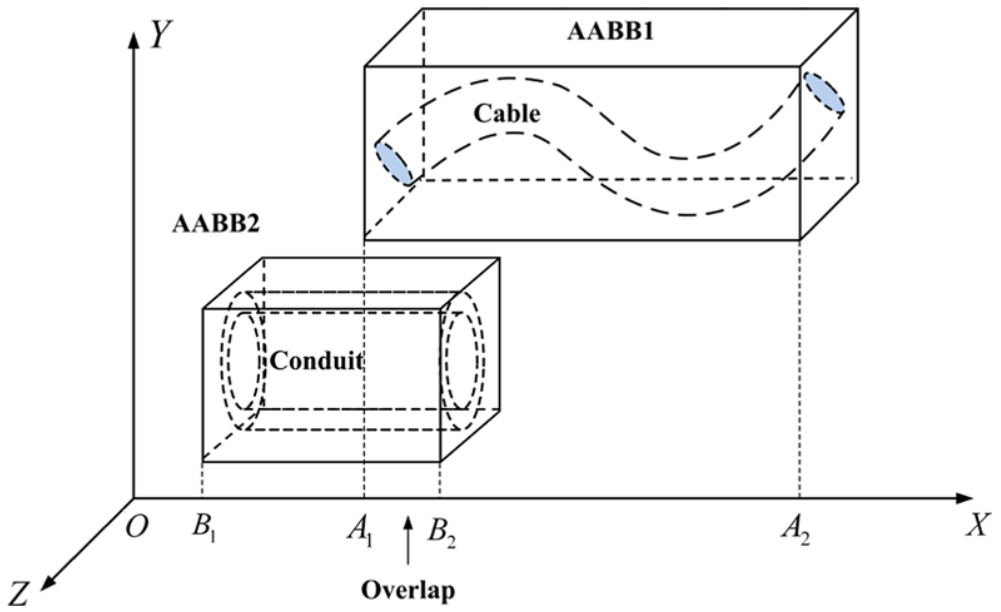

Fig. 5 Schematic of axis aligned bounding box

\subsection{Acceleration technique of contact check}

Figure 4 illustrates the contact between one cable element and one straight segment of the conduit. In an actual computation, the cable and the conduit are divided into many elements and straight segments for the sake of the computation precision. For contact detection, the check will be processed between any cable element and any straight segment of the conduit. Obviously, the efficiency of computation is very low.

In this section, a precontact searching algorithm of the axis aligned bounding box (AABB) [18] is employed. As shown in Fig. 5, the axis aligned bounding box is a rectangular cuboid, whose edges are aligned with (parallel to) the axes $(\mathbf{X}, \mathbf{Y}$, and $\mathbf{Z}$ ) of the coordinate system.

In Fig. 5, the cable element is enclosed in the $\mathrm{AABB}$ one and the AABB two encloses one of the segments of the conduit. The line $A_{1} A_{2}$ is the projection of the AABB one onto the $X$ axis. The line $B_{1} B_{2}$ is the projection of the AABB two onto the $X$ axis. If there is no overlap between the projection line $A_{1} A_{2}$ and the projection line $B_{1} B_{2}$, the AABB one does not intersect with the AABB two. It means the cable enclosed in the AABB one does not contact with the current segment of the conduit enclosed in the AABB two. Otherwise, contact maybe occurs. A further inquiry of the overlap between the projection lines of the two AABBs onto the other two axes is required. Obviously, a maximum of three attempts can detect contact or not, so the speed of contact test has been improved.

\subsection{Cable tension model}

Based on the analysis presented in the above text, the tensile force $N$ in the arbitrary cross section of the cable element can be decided by the following differential algebraic equations (DAEs):

$$
\left\{\begin{array}{l}
\mathbf{M} \ddot{\mathbf{q}}=\mathbf{Q}_{q}+\mathbf{C}_{q}^{T} \boldsymbol{\lambda}_{q} \\
\mathbf{C}(\mathbf{q}, \dot{\mathbf{q}}, t)=0 \\
N=E A \varepsilon
\end{array}\right.
$$


Fig. 6 Cable pulled out the deviated conduit

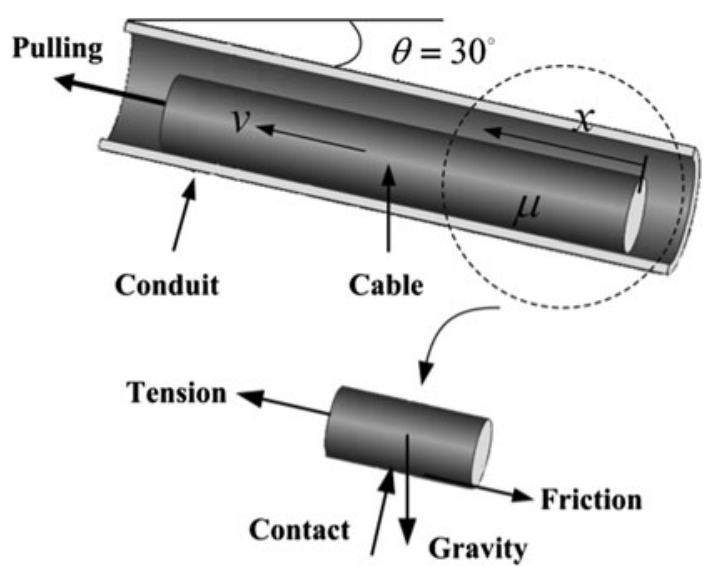

where $\mathbf{q}$ is the generalized coordinate vector of the system, $\mathbf{M}$ is the mass inertia of the system, $\mathbf{Q}_{q}$ is the generalized force vector of the outer forces applied on the system. In this problem, the outer applied forces of system mainly include the gravity of the cable and the contact force between the cable and the conduit, etc. $\mathbf{C}$ denotes the constraints of the system, for example, the velocity constraint exerted by the pulling rig on the moving cable during the cable installation.

Many numerical methods [19, 20] can be use to solving Eq. (24). In this paper, a backward difference formulation [21] is applied. After obtaining the solution of Eq. (24), one can calculate the tension in an arbitrary cross section of the cable element.

\section{Numerical examples}

Four numerical examples examined in this section are used to illuminate the effectiveness and validity of the presented model in problems of predicting the tensile force of the cable in installation of the cable.

In all the examples, the conduits are assumed to be rigid and fixed in the ground. The cables and the conduits have the circular cross sections with radius $2.5 \mathrm{~mm}$ and $3.5 \mathrm{~mm}$, respectively. In simulation, the cables are discretized by enough finite elements and the conduits are divided into enough straight segments. The cables have mass density $\rho=1000 \mathrm{~kg} / \mathrm{m}^{3}$, Young's modulus $E=10^{9} \mathrm{~Pa}$ in all cases. The damping coefficient of the cable element is 0.005 in this paper. The contact stiffness and contact damping between the cable and the conduit are $1000 \mathrm{kN} / \mathrm{m}$ and $1 \mathrm{kN} \mathrm{s} / \mathrm{m}$, respectively. The nonlinear contact exponent is 1.1 .

\subsection{Example 1}

The cable is pulled in a deviated conduit with inclined angle $\theta=30^{\circ}$, as shown in Fig. 6 . The cable has total length of $50 \mathrm{~m}$, which is divided into 40 elements in this example. The cable pulling ring is supposed to drag the cable at its top end, which is not drawn in Fig. 6. Under the action of the pulling rig, the top end of the cable is assumed to move along the conduit at constant speed of $0.1 \mathrm{~m} / \mathrm{s}$. The tension of the cable is a result of the friction of the cable rubbing on and contacting with the conduit wall. The friction coefficient $\mu$ is 0.3 . To model the contact, 400 check points are used in each element of the cable. When the cable 
Fig. 7 The distribution of the tension along the cable

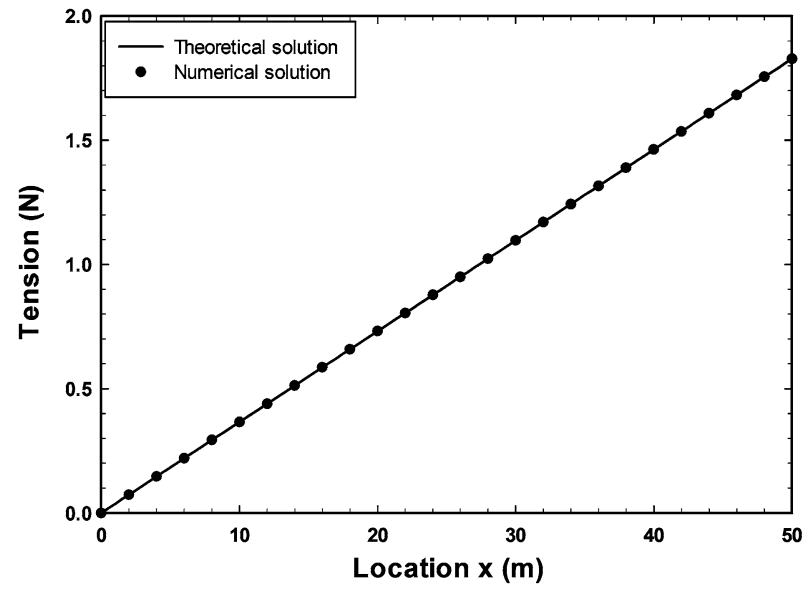

is in the steady-state motion, the tensile force $T$ in the arbitrary cross section of the cable at $x$ is theoretically decided by

$$
T=\rho A g(\mu \cos \theta+\sin \theta) x
$$

where $g$ is the gravity acceleration and $g=9.8 \mathrm{~m} / \mathrm{s}^{-2}$.

Figure 7 shows the distribution of the tension of the cable along its length at the steady state of the system. The plot labeled by the dot points denotes the numerical results and the solid line represents the theoretical results. Clearly shown in Fig. 7, the numerical results approximate well with the theoretical results.

\subsection{Example 2}

In this example, the gravity is ignored. The conduit with a 90-degree bend, as shown in Fig. 8, is considered. The radius of bend is $0.5 \mathrm{~m}$. The friction coefficient $\mu$ between the cable and the wall of conduit is 0.2 . Two tensile forces $T_{1}$ and $T_{2}$ are assumed to be applied at the top end and the low end of the cable, respectively. Both the directions of $T_{1}$ and $T_{2}$ are aligned with the tangent of the centerline of the conduit at its corresponding end. Under the action of the two tensile forces $T_{1}$ and $T_{2}$, the cable is assumed to be pulled out of the conduit from the top end, slowly. The tensile force $T_{2}$ is $150 \mathrm{~N}$.

As the cable-conduit system is at steady state, the magnitude of the tensile force $T_{1}$ can be theoretically given by

$$
T_{1}=T_{2} e^{\mu \beta}=205.36616 \mathrm{~N}
$$

where $\beta$ denotes the helical angle and is equal to $\pi / 2$ in this numerical example. The numerical result for the tensile force $T_{1}$ is about $205 \mathrm{~N}$, seen in Fig. 9. There is a good agreement between the theoretical solution and the numerical solution. In simulation of this example, the cable is discretized by 40 elements and the conduit is divided into 100 straight segments. Two hundred check points are used in each element of the cable.

\subsection{Example 3}

In this example, we consider a complex conduit system-helical conduit, seen in Fig. 10. In numerical simulation, the gravity is also ignored and the number of the coils of the helical 
Fig. 8 Cable pulled out the conduit with a 90 degree bend
Fig. 9 The tension $T_{1}$ and $T_{2}$ of the cable in the conduit with a 90 degree bend

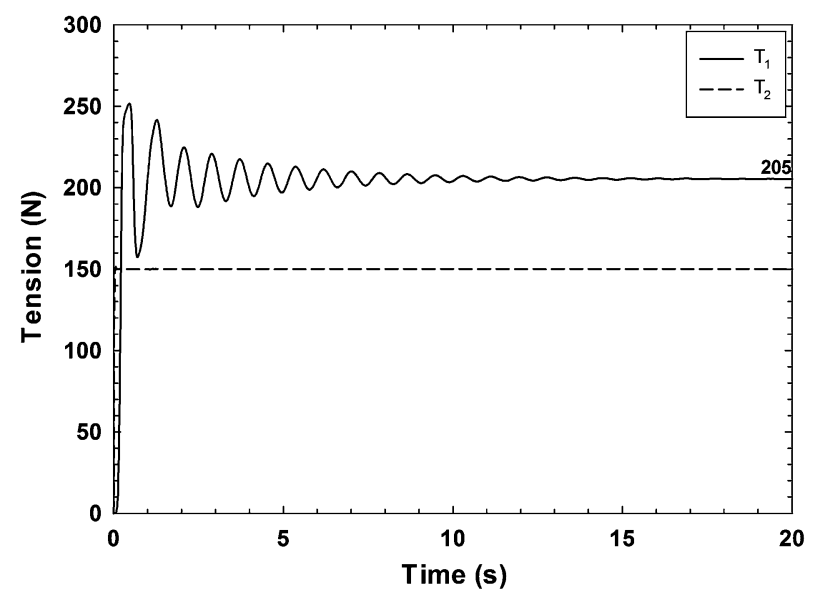

Fig. 10 Cable pulled out a helical conduit

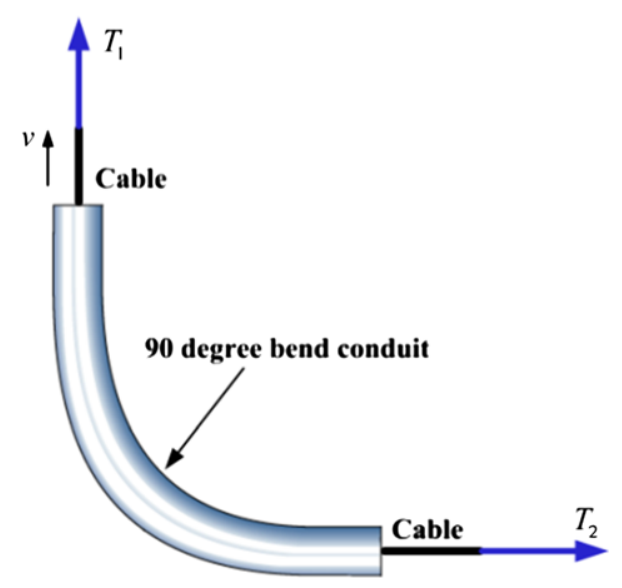

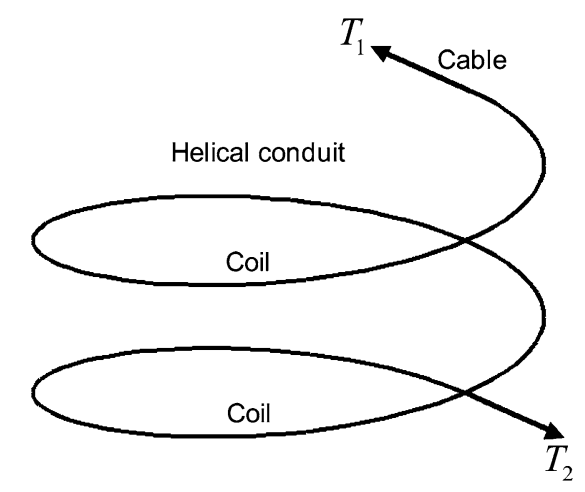

conduit is two. Here, the mean radius of the conduit is $1 \mathrm{~m}$. The cable is discretized by 80 elements. The conduit is divided into 200 straight elements. Five hundred check points are used in each element of the cable. The friction coefficient $\mu$ between the cable and the wall of conduit is 0.1 . The tensile force $T_{1}$ is $40 \mathrm{~N}$. When the cable is slowly pulled out 
Fig. 11 The tension $T_{1}$ and $T_{2}$ of the cable in the helical conduit

Fig. 12 The required force $T_{1}$ of the cable pulled in the helical conduit with different coils
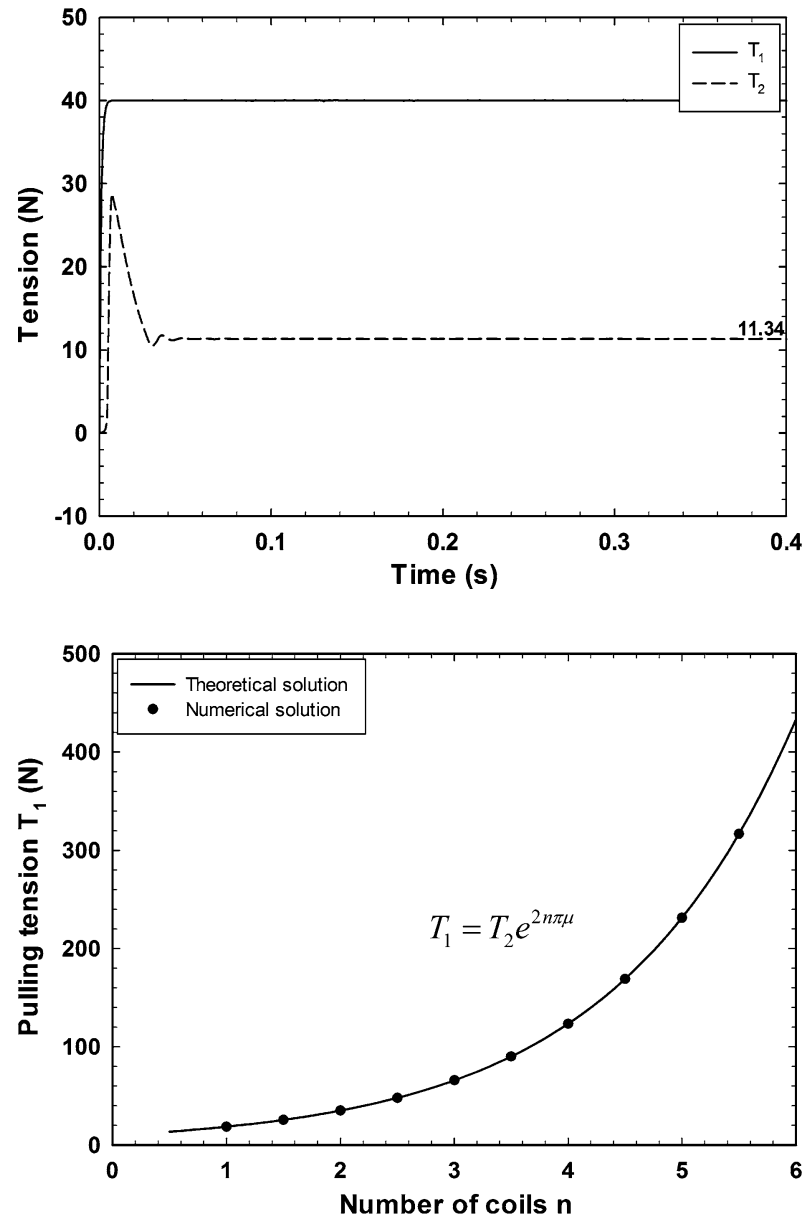

the conduit from the top end, the tension $T_{2}$, which can be theoretically decided by the law defined in Eq. (26), is $11.3844 \mathrm{~N}$. The helical angle $\beta$ is $4 \pi$ in this numerical example.

Figure 11 shows the change of the tension of the cable at the two ends against with time. The plots in Fig. 11 illustrate the tensile force $T_{2}$ is $11.34 \mathrm{~N}$ as the cable-conduit system is in steady state. The relative error between the numerical solution and theoretical solution is only $0.39 \%$.

Moreover, we investigate the required pulling force as the cable pulled out the above helical conduit for different coils. The tension $T_{2}$ of the cable at the low end is $10 \mathrm{~N}$. The other parameters are the same as the ones used in this example. As the cable pulled slowly in the conduit, the change of the required pulling force $T_{1}$ with the number of the coils of the helical conduit accords with the exponential law, which is plotted by the dots in Fig. 12.

\subsection{Example 4}

Figure 13 shows a complex spatial conduit system. It consists of three straight segments and three bending segments. In this case, we concern on the pulling force when the cable is pulled out of the conduit. Obviously, the theoretical solution for this problem is not 


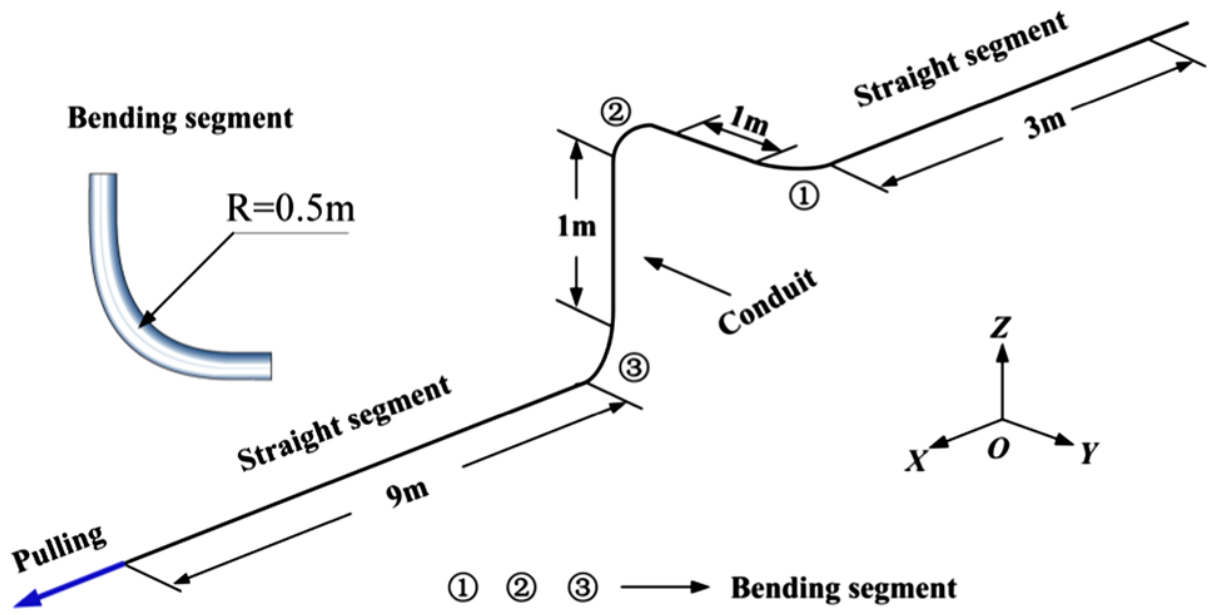

Fig. 13 Cable pulled out of the spatial conduit

Fig. 14 Comparison of the cable pulling forces in two methods

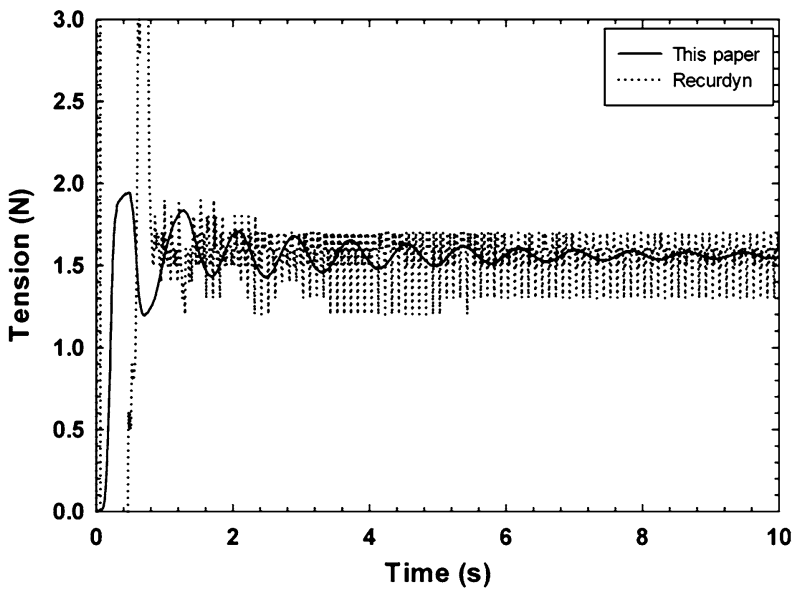

found. To validate the results (depicted by solid line in Fig. 14) obtained from the presented method, we use Recurdyn (a famous dynamic solver) to solve this problem and make a comparison between these two results. The corresponding results obtained from Recurdyn are plotted by the dotted line in Fig. 14. As shown in Fig. 14, the two results are approximated comparatively well with each other.

In simulation, the cable is equally divided into 100 elements. The bend segment of the conduit is divided into 80 straight segments. Eight hundred check points are set in each element of the cable. Table 1 accounts for the computational efficiency of different methods in this example.

\section{Conclusions}

This paper focuses on the tensile force of the cable in installation. A computational model based on multibody system dynamic formulation for predicting the cable tension is pre- 
Table 1 Comparison of computational efficiency for different methods

\begin{tabular}{ll}
\hline Method & CPU time (min) \\
\hline Our method with AABB test & 4.6 \\
Our method without AABB test & 63.2 \\
Recurdyn & 180
\end{tabular}

sented. Compared with conventional models, the presented model is general and has capacity of predicting the cable tension in a complex conduit with arbitrary shape. The presented examples correlate very well with theoretical solutions when available and are very efficient when compared with commercial software.

Open Access This article is distributed under the terms of the Creative Commons Attribution License which permits any use, distribution, and reproduction in any medium, provided the original author(s) and the source are credited.

\section{References}

1. Rifenburg, R.C.: Pipe-line design for pipe-type feeders. Trans. Am. Inst. Electr. Eng. (1953). doi:10. 1109/AIEEPAS.1953.4498766

2. Smith, D.G.: A general method for the calculation of pipe cable pulling forces. IEEE Trans. Power Appar. Syst. PAS-100(1), 360-368 (1981). doi:10.1109/TPAS.1981.316864

3. Iordanescu, M., Tamowski, J.: Pullflex —new software for duct-cable pulling forces. IEEE Trans. Power Deliv. 11(2), 676-682 (1996). doi:10.1109/61.489322

4. Bullard, W.R., Pettee, A.D., Rhodes, G.L.: 115 kV High-pressure oil-filled pipe cable installation at New Orlean La. Trans. Am. Inst. Electr. Eng. 67(1), 475-486 (1948). doi:10.1109/T-AIEE.1948.5059697

5. Buller, F.H.: Pulling tensions during cable installation into ducts or pipes. Gen Electr Rev 52(8), 21-23 (1949)

6. Bosworth, D.W.: Cable pulling tension in ducts calculated by simplified method. Electrical World 152$153(1954)$

7. Imai, T., Kikuchi, K., Takada, N., Ishizu, K.: Cable installation technique challenging sharp difference in land elevation. IEEE Trans. Power Appar. Syst. PAS-90(4), 1883-1893 (1971). doi:10.1109/TPAS. 1971.293183

8. Schwab, A.L., Meijaard, J.P.: Comparison of three dimensional flexible beam elements for dynamic analysis classical finite element formulation and absolute nodal coordinate formulation. J. Comput. Nonlinear Dyn. 5(1), 1-10 (2010). doi:10.1115/1.4000320

9. Von Dombrowski, S.: Analysis of large flexible body deformation in multibody systems using absolute coordinates. Multibody Syst. Dyn. 8(4), 409-432 (2002). doi:10.1023/A:1021158911536

10. Gerstmayr, J., Shabana, A.A.: Analysis of thin beams and cables using the absolute nodal coordinate formulation. Nonlinear Dyn. 45(1-2), 109-130 (2006). doi:10.1007/s11071-006-1856-1

11. Sopanen, J.T., Mikkola, A.M.: Description of elastic forces in absolute nodal coordinate formulation. Nonlinear Dyn. 34(1-2), 53-74 (2003). doi:10.1023/B:NODY.0000014552.68786.bc

12. Gerstmayr, J., Irschik, H.: On the correct representation of bending and axial deformation in the absolute nodal coordinate formulation with an elastic line approach. J. Sound Vib. 318, 461-487 (2008)

13. Garcia-Vallejo, D., Valverde, J., Dominguez, J.: An internal damping model for the absolute nodal coordinate formulation. Nonlinear Dyn. 42(4), 347-369 (2005). doi:10.1007/S11071-005-6445-1

14. Kerkkänen, K.S., García-Vallejo, D., Mikkola, A.M.: Modeling of belt-drives using a large deformation finite element formulation. Nonlinear Dyn. 43(3), 239-256 (2006)

15. Lankarani, H.M., Nikravesh, P.E.: Continuous contact force models for impact analysis in multibody systems. Nonlinear Dyn. 5(2), 193-207 (1994)

16. Fee, J.M.: A new cable pulling friction measure method and results. IEEE Trans. Power Deliv. 7(2), 681-686 (1992). doi:10.1109/61.12706

17. Fee, J.M.: A pulling tension calculation program which allows coefficient of friction to vary continuously with cable sidewall pressure. IEEE Power Engineering Society, Transmission and Distribution Conference 314-316 (1994). doi:10.1109/TDC.1994.328444 
18. Li, C.F., Fenq, Y.T., Qwen, D.R.J.: SMB: collision detection based on temporal coherence. Comput. Methods Appl. Mech. Eng. 195(19-22), 2252-2269 (2006). doi:10.1016/j.cma.2005.05.003

19. Fox, B., Jennings, L.S., Zomaya, A.Y.: Numerical computation of differential algebraic equations for nonlinear dynamics of multibody systems involving contact forces. J. Mech. Des. 123(2), 272-281 (2001). doi:10.1115/1.1353587

20. Simeon, B.: Numerical analysis of flexible multibody systems. Multibody Syst. Dyn. 6(4), 305-325 (2001). doi:10.1023/A:1012096631385

21. Hairer, E., Wanner, G.: Solving Ordinary Differential Equations II: Stiff and Differential Algebraic Problems. Springer, Berlin (1996) 